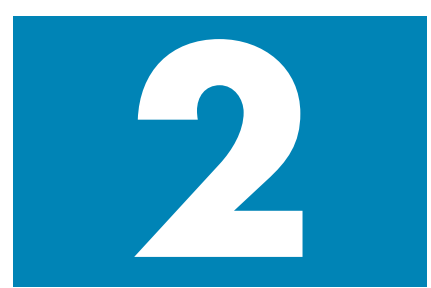

\title{
The examination and recording of the occlusion: why and how
}

\author{
S. J. Davies, ' and R. M. J. Gray, ${ }^{2}$
}

\author{
Before presenting 'how' the examination and recording of the \\ occlusion may be achieved, some attempt should be made to \\ justify 'why' it is necessary. It may appear to be a strange way of \\ justifying the need to examine the patient's occlusion, but this \\ will initially involve a study of the influences of mandibular \\ movements.
}

In this part, we will discuss:

- How an understanding of mandibular locomotive systems compels dentists not to ignore occlusion

- How the occlusion can be simply and quickly examined and recorded
${ }^{*} G D P, 73$ Buxton Rd, High Lane, Stockport SK6 8DR; P/T Lecturer in Dental Practice, University Dental Hospital of Manchester, Higher Cambridge St., Manchester M15 6FH; ${ }^{2}$ Honorary Fellow, University Dental Hospital of Manchester, Higher Cambridge St., Manchester M15 6FH ${ }^{*}$ Correspondence to: Stephen Davies, 73 Buxton Rd, High Lane, Stockport SK6 8DR

email:stephen.j.davies@man.ac.uk REFEREED PAPER

(C) British Dental Journal

2001; 191:291-302
$\mathrm{T}$ he mandible moves, relative to the maxilla, by virtue of two influences. Firstly, locomotive forces are provided by the muscles under the control of the nervous system: neuromuscular control. Secondly, there are two hard tissue guidance systems: these are the temporomandibular joints and the occlusal surfaces of the teeth.

\section{Neuromuscular control}

The muscles

Mandibular muscles

(The term mandibular muscles is preferable to masticatory muscles in the same way that 'leg muscles' is a more embracing term than 'walking muscles').

Within the articulatory system the muscles have been simply expressed as the 'motors' Whereas it is the 'hard' occlusal surfaces of the teeth and articulatory surfaces of the bones which provide the guidances of mandibular movement, it is muscles which provide the locomotive force to move the mandible during function and parafunction. The muscles which are joined to the mandible and are therefore responsible for its movement are singularly and collectively immensely complicated motive entities.

It may be because anatomy was the first medical science, that the function of a muscle has historically been decided purely by an analysis of its origin and insertion. This is dangerously simplistic and has sometimes resulted in a mandibular muscle having been labelled as simply as an 'opening' or 'closing' muscle. It takes no account of the complex antagonistic and synergistic interrelations of muscle function, which are responsible for supplying the motive power of mandibular movement. Electromyographic recording when linked to either simple observation or sophisticated jaw tracking systems offers an enhanced understanding of the functions of the mandibular muscles. The lateral pterygoid muscle offers a good example of how the understanding of its function was enhanced by electromyography, beyond the simple anatomical assumptions previously held. ${ }^{1}$

Individual mandibular muscles

The masseter muscle

Anatomy: The masseter originates from the zygomatic arch, inserts into the outer surface of the angle of the mandible and comprises superficial, intermediate and deep parts.

Function: Its principal action is to elevate the mandible, so closing the jaws; it is also an accessory muscle in mandibular protrusion.

Parafunction: It is active in tooth clenching and is the most frequently affected muscle by this parafunctional habit.

\section{The temporalis muscle}

Anatomy: This is a large, fan shaped muscle arising from the lateral aspect of the skull in the temporal fossa and converges to a tendinous insertion, which, running below the zygomatic arch, inserts into both the coronoid process and anterior border of the ascending ramus of the mandible.

It is significant that the orientation of the muscle fibres varies greatly:

- The posterior fibres run almost horizontally forwards

- The anterior fibres run vertically.

- The intermediate fibres have varying degrees of orientation.

Function: The action of this muscle depends upon which fibres are contracting:

- The anterior fibres raise the mandible when the mouth is closing

- The horizontal posterior fibres retract the mandible. The horizontal fibres of the tem- 


\begin{tabular}{l} 
Mandibular \\
movement \\
- Controlled by the \\
Neuromuscular \\
system \\
- Influenced by \\
two hard guidance \\
systems: \\
Temporomandibular \\
joints, and \\
Occlusal surfaces of \\
teeth \\
\hline
\end{tabular}

Occlusal examination
- Against the
benchmark of
ideal occlusion
- Three essential
questions

poralis muscle are the only muscle fibres that retract the mandible; no other muscle performs this function.

Parafunction: The temporalis muscle is the most frequently tender muscle in bruxists

\section{Lateral pterygoid muscle}

Anatomy: This is a muscle which has two heads and, it is now thought, two insertions. The smaller superior head arises predominantly from the infra-temporal surface of the greater wing of the sphenoid and inserts into the anterior part of the intra-articular disc and capsule, while the larger, inferior head arises from the lateral surface of the lateral pterygoid plate and inserts into the neck of the mandible just below the condyle.

Function: While it is accepted that there is some overlap of activity of the two heads, the superior pterygoid is predominantly active during clenching and is thought to stabilise the condyle disc assembly. The inferior pterygoid is active during mouth opening and draws the condyle and disc forwards and down the slope of the articular eminence.

Therefore, when both right and left pterygoid muscles act together, mouth opening and mandibular protrusion occurs, but when one muscle contracts the condyle on that side is drawn forwards and the mandible pivots around the opposing condyle and the chin, therefore, moves towards the opposite side.

Parafunction: This active/passive cycle is altered, however, in parafunction; when it has been demonstrated that there is an overall disruption of the pattern with both heads (or 'muscles' if superior and inferior bellies are considered to be separate muscles) showing a marked and simultaneous increase in activity. This can cause pain in the pre-auricular region. In addition, it may possibly lead to disc displacement, and the patient may develop TMJ clicking and locking.

\section{The medial pterygoid muscle}

Anatomy: The bulk of this muscle originates from the area between the two pterygoid plates; there is, however, a small, more superficial head arising from the maxillary tuberosity. These two heads fuse and the muscle passes posteriorly and laterally downwards to insert into the inner aspect of the angle of the mandible. The orientation of the fibres parallels that of the anterior fibres of the masseter muscle.

Function: The action of the medial pterygoid muscle is to elevate the mandible and it is also active during protrusion and lateral mandibular movement.

Parafunction: It is not a muscle that can reliably be palpated for tenderness, nor tested by resisted movement, and so its involvement in parafunction can only be surmised. It may become hypertonic in patients who parafunction in extreme mandibular movement (eg the crossover position)

\section{Digastric muscle}

Anatomy: This muscle has two parts - the anterior and posterior bellies. They are connected by a tendon which passes through a fibrous loop on the upper border of the hyoid bone. The posterior belly arises from the mastoid notch and the anterior belly is inserted into the mandible near the symphysis. This insertion into the mandible qualifies it for inclusion as a 'mandibular muscle'.

Function: When the hyoid bone is fixed (by the infrahyoid muscles) the action of this muscle is to assist the lateral pterygoid muscle in opening the mouth. Its action is therefore to depress mandible. In the action of swallowing, the hyoid bone is raised by both right and left digastric muscles contracting together.

Parafunction: Tenderness in this muscle is frequently encountered in patients who brux or clench on their anterior teeth and manifests as pain behind the ascending ramus or under the body of the mandible.

\section{Mylohyoid muscle}

Anatomy: This thin sheet of muscle arises from the whole length of the mylohyoid line on the inner aspect of the mandible. The fibres meet in the median raphe, which inserts into the body of the hyoid bone. This muscle separates the submandibular and sublingual regions. Posteriorly the muscle has a free border.

Function: The action of this muscle is to raise the hyoid bone and the tongue during swallowing.

\section{Suprahyoid, infrahyoid and cervical muscles}

Function and parafunction: The reason why a brief consideration of the function and parafunction of these muscles should be discussed as part of the subject of occlusion is that claims are made that occlusion can have an effect on the wider musculo-skeletal system, even the lumbar spine. This supposition is based upon a consideration of head posture.

The hyoid bone is attached to the mandible by the suprahyoid muscles. The infrahyoid and suprahyoid muscles by stabilising the hyoid bone enable the suprahyoid muscles to be tangentially involved in mandibular movement. Head posture is also affected by the action of these muscles, as shown by the fact that the head moves slightly back as the mandible opens. Conversely, head posture could potentially affect the function and health of these muscles. This may provide an explanation for an association between head posture and myalgia of the head and neck muscles.

Similarly the cervical muscles are largely 
responsible for head posture; and there maybe an association between some TMD and symptoms of the cervical spine.

This hypothesis leads to the question, should dentists adjust the 'occlusion' to treat head and neck muscle pain?

It is right that dentists, together with other health professionals, constantly examine their understanding of the biomechanics of interlocking locomotive systems. It is obviously true that the occlusion of teeth is a part of this system, although a small part. But there is no evidence that TMD follows the simple model of single cause, single diagnosis and single treatment and certainly there is no proof the occlusion is that single cause! We, therefore, feel that unless such a causal relationship is established, treatment to the teeth or permanent change of the position of the mandible is not indicated in pursuit of either a TMD or other musculo-skeletal disorder. In summary, 'thought, but not action' is appropriate.

\section{Neural pathways}

The mandible is controlled not only as a result of voluntary movement, but also by reflexes, most notably a jaw closing reflex and jaw opening reflex. The jaw-closing reflex protects the mandible and associated structures during violent whole body movement; it can result in damage to the teeth, especially if the occlusal contacts are not in the long axis of the root. The jaw opening reflex is to protect the teeth during sudden mastication of a hard object and to protect the lips, cheeks and tongue during mastication. These voluntary and involuntary movements are controlled by the central and autonomic nervous systems via sensory and motor nerves.

There is input to these systems from peripheral receptors and the higher centres, and also specific proprioceptors. These proprioceptors are not only situated in deep muscle, as in other human locomotive systems, but also in the periodontal ligaments. It is the presence of the periodontal proprioceptors which makes the articulatory system unique amongst human locomotive systems. No other locomotive system incorporates teeth which, because they are attached to proprioceptive receptors, means that if the movements within this locomotive system results in teeth touching (either in function or parafunction) then these proprioceptors are stimulated. As a consequence there is the potential for any change in a patient's occlusion, by routine dentistry, to 'be sensed' by the patient's nervous system. It is because of this consideration that dentists cannot ignore the effect of changing the occlusion when providing routine care.

\section{Fig. I Illustration of the direction of mandibular movement}

The direction of movement of the NWS condyle is forwards, downwards and medially (FDM). It is defined against the horizontal plane $(H)$ by the condylar angle and against the vertical plane $(\mathrm{V})$ by the Bennet angle.

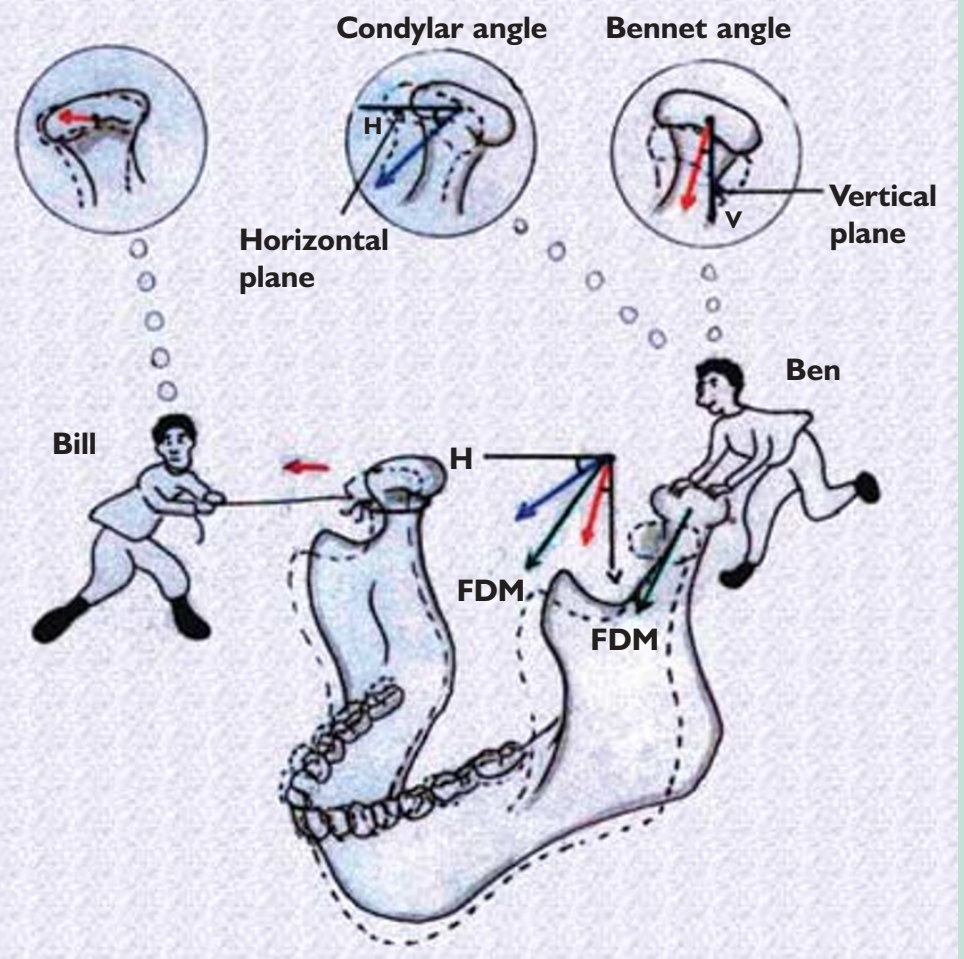

This drawing is not designed to represent the muscles that are responsible for mandibular movement. Rather it is designed to illustrate how the NWS condyle moves forwards, downwards and medially; whereas the WS condyle undergoes an immediate non-progressive lateral movement. It is known as immediate side shift (ISS) or Bennet movement. The mandible moves as if Bill pulls it directly towards him, and Ben pushes it downwards, forwards and slightly towards Bill's side.

\section{The guidance systems}

There are two systems that provide hard guidance during mandibular movements. These are classified as the posterior and the anterior guidance systems but they are interrelated guidances and are part of one system. An understanding of them is important to dentists and relevant to this work, because occlusion provides one of them.

\section{Posterior guidance}

The temporomandibular joint is made up of the head of the condyle, the intra-articular disc and the glenoid fossa; and it provides the posterior guidance system of the mandible. During a lateral excursion of the mandible, the principle movement within the TMJs is on the nonworking side (NWS).

The head of the condyle on the non-working side moves: forwards, downwards and medially. 
- The angle of downwards movement is known as the 'condylar angle'

- The angle of medial movement is known as the 'Bennet angle'.

There is, however, a movement of the condyle on the working side as this is described as 'immediate side shift', or 'Bennet movement'. This movement is immediate, non progressive and lateral.

It is the mechanical consequence of the working side condyle being joined up to the non-

\begin{tabular}{l} 
Fig. 2 \\
Static occlusion \\
Does CO occur in CR? \\
If not.. prem contact in CR? Roughly: \\
Exactly: \\
Direction of slide from CR to CO: \\
\hline
\end{tabular}
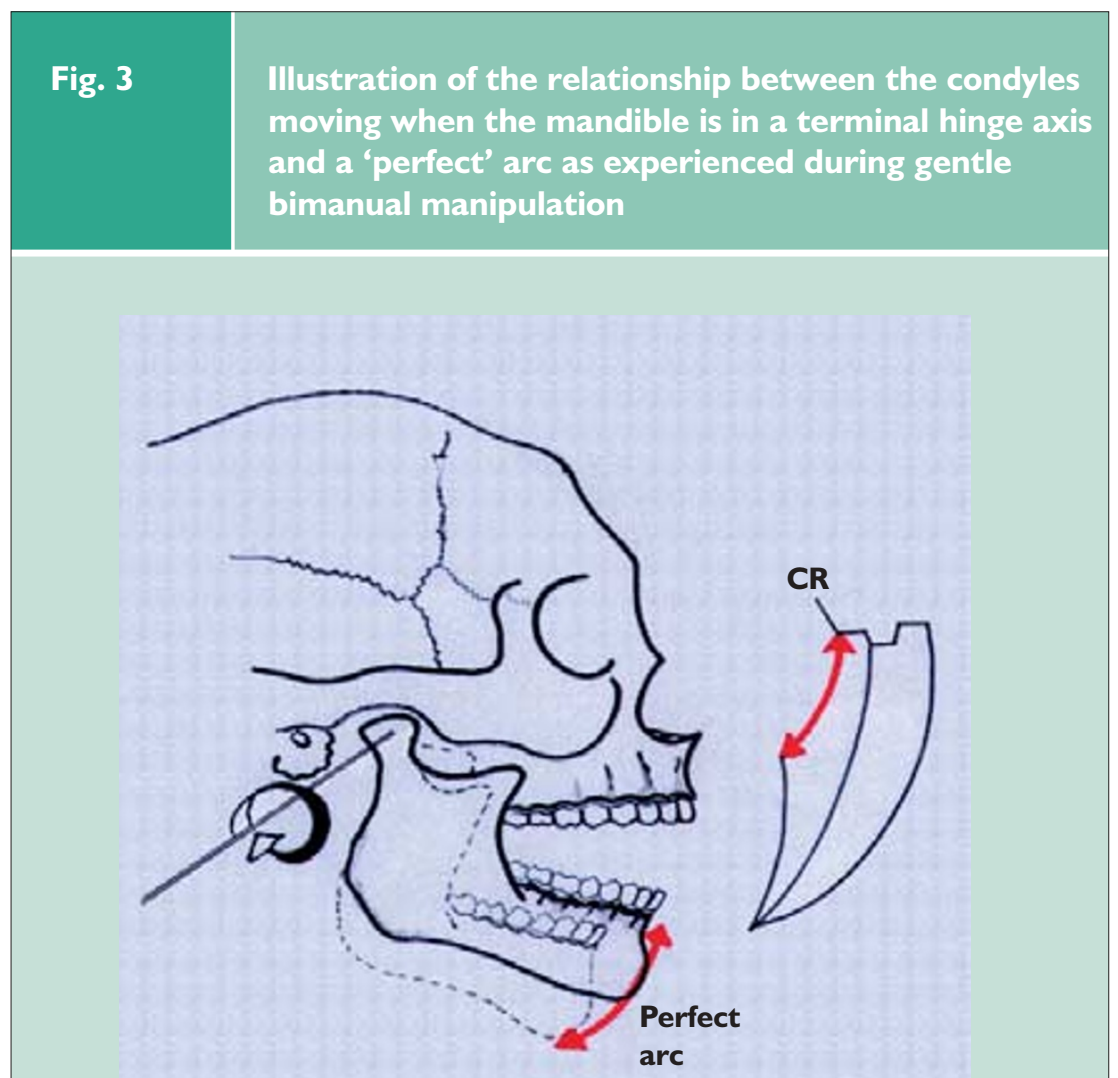

working side condyle, whilst the latter is being pulled by muscles.

Figure 1 provides an illustration on mandibular movement during a right lateral excursion.

\section{Anterior guidance}

The anterior guidance system of the mandible is provided by which ever teeth touch during the excursive movements of the mandible, whilst teeth are in contact. These contacts are known as the dynamic occlusion.

Whilst it is generally considered to be more ideal if these contacts occur at the front of the mouth (ie as far away as possible from the posterior guidance system), it must surely be that even the back teeth may provide the anterior guidance of the mandible, because all teeth are anterior to the temporomandibular joints. The term 'anterior guidance' is often used in restorative textbooks to mean anterior guidance of the mandible which is exclusively on front teeth. This would be better described as ideal anterior guidance'. Ideal occlusion as a concept is described elsewhere in this work.

\section{Relevance of a study of guidance systems to occlusion}

The reason why dentists need to concern themselves with mandibular movements and the influences that control them is that most dental treatment involves the occlusal surfaces of teeth: that is to say that dentists inevitably are changing one of the guidance systems of mandibular movement.

There is little evidence to suggest that a change in occlusion will precipitate morphological changes within the joint. It would appear, therefore, that the most likely adaptation in the 'system' is that which can occur in the teeth and their supporting structures. These 'adaptations' are tooth wear, tooth movement and fracture.

\section{Articulatory system: occlusal harmony?}

How to determine what is an occlusion that conforms to the TMJ may appear to be an impossible question to answer; in reality the solution is very simple.

Firstly a competent examination of the whole of the articulatory system must be carried out. This will include the TMJs and the supporting muscles to determine whether there is any temporomandibular disorder present.

If there is a TMD present, then the first clinical decision to be taken, with the patient's involvement, will be whether or not to treat it before providing any other treatment.

Next, the patient's pretreatment occlusion must be examined, because, dependent upon the type and extent of the dental treatment necessary, the next essential treatment plan- 
ning decision will be: 'can the treatment be provided without changing the patient's preexisting occlusion?'

If it can, then the conformative approach is to be adopted. If it cannot, then it will be safer if the new occlusion is going to be more ideal rather than less ideal to the rest of the masticatory system than the pretreatment occlusion was. This is known as the 'reorganised approach'.

Either of these two approaches would constitute good occlusal practice. It would be bad occlusal practice accidentally or otherwise to make the occlusion a different or less ideal one.

This introduction has brought us to the conclusion that an examination of the patient's pre-treatment occlusion is essential.

\section{Examination of the occlusion (Fig. 8)}

\section{Introduction}

As stated in the previous section, the principle function of the concept of ideal occlusion in everyday dentistry is to provide a benchmark against which any patient's occlusal pattern can compared. This does not infer that the provision of an ideal occlusion is the treatment objective for that particular patient nor for patients in general.

\section{The three question examination}

\section{Question 1. Does Centric Occlusion occur in}

Centric Relation? (Fig. 2)

Centric relation describes a relationship between the two jaws, it has nothing to do with teeth; it is not an occlusion. When the head of the condyle is moving purely in the rotational phase of its movement, then the mandible is in a terminal hinge axis. This concept provides one of the three pillars of the definition of centric relation that is given in the previous section. If the head of the condyle is the stationary centre about which the mandible is rotating, then the mandible, during this phase of movement will describe an arc. Whereas if the mandible is not in terminal hinge axis, then the head of the condyle will not be purely rotating, because there will be an element of translation; and as a consequence of the fact that the head of the condyle is, therefore, not stationary (in the antero-posterior plane) the mandible will not describe an arc.

Manipulation of patient to find the Centric Relation It is feeling that the patient's mandible is describing a perfect arc during manipulation that gives the experienced operator the confidence that the terminal hinge axis of the mandible (Fig. 3) has been found. It is certain that in reality the mandible is not describing a perfect arc because unlike on an articulator the condyle is never a perfect sphere and the

\section{Static occlusion}

Freedom in Centric Occlusion?

Fig. 5 Occlusal examination - question 3

\section{Dynamic occlusion}

RHS LHS
Won-working side interferences
Working side interferences
$\begin{aligned} & \text { Crossover position } \quad \text { NWS Int } \\ & \text { Canine guidance }\end{aligned}$
Group function

glenoid fossa is never a hemisphere. There is, however, one important test that provides confidence that Centric Relation has been found. This test is based upon the fact that because the Centric Relation is a jaw relationship not guided by teeth nor by the patient's muscles but by the operator arcing the mandible in its terminal hinge axis towards the maxilla, and that the end point of this arc will be consistent. The end point of this arc occurs when the first teeth touch and this is known as the premature contact in the Centric Relation. Centric Relation is the only 'Centric' which is consistent. It is the fact that the Centric Relation has been found to be consistent in any one patient irrespective of time or operator that makes it so significant as a concept. It may be that the end point of the closing arc of the mandible whilst it is in terminal hinge axis (Centric Relation) is not a premature contact but rather an even contact of all of the teeth (Maximum Intercuspation). In this case the Centric Relation and Centric Occlusion coincide. If, however, as is usually 
- Operator seated comfortably

- Patient is supine, at elbow height

- Patient's neck extended slightly

- Use both hands

- Thumbs over mental symphasis

- Fingers on the lower border of mandible, not sublingually

- Ask the patient to relax their jaw and let you open their mouth

- Slowly and gently arc mandible up and down with minimum force

- Only the patient's mandible should be moving

- Slowly increase the upward component of each arc, until premature contact is reached

- Ask the patient to put a finger in the air when they feel first contact

- Repeat at least three times

- Ask patient: It is the same each time?

- Ask patient to use raised finger to point where they feel first contact

- Ask patient to squeeze teeth together from premature contact, so as to note the direction of slide
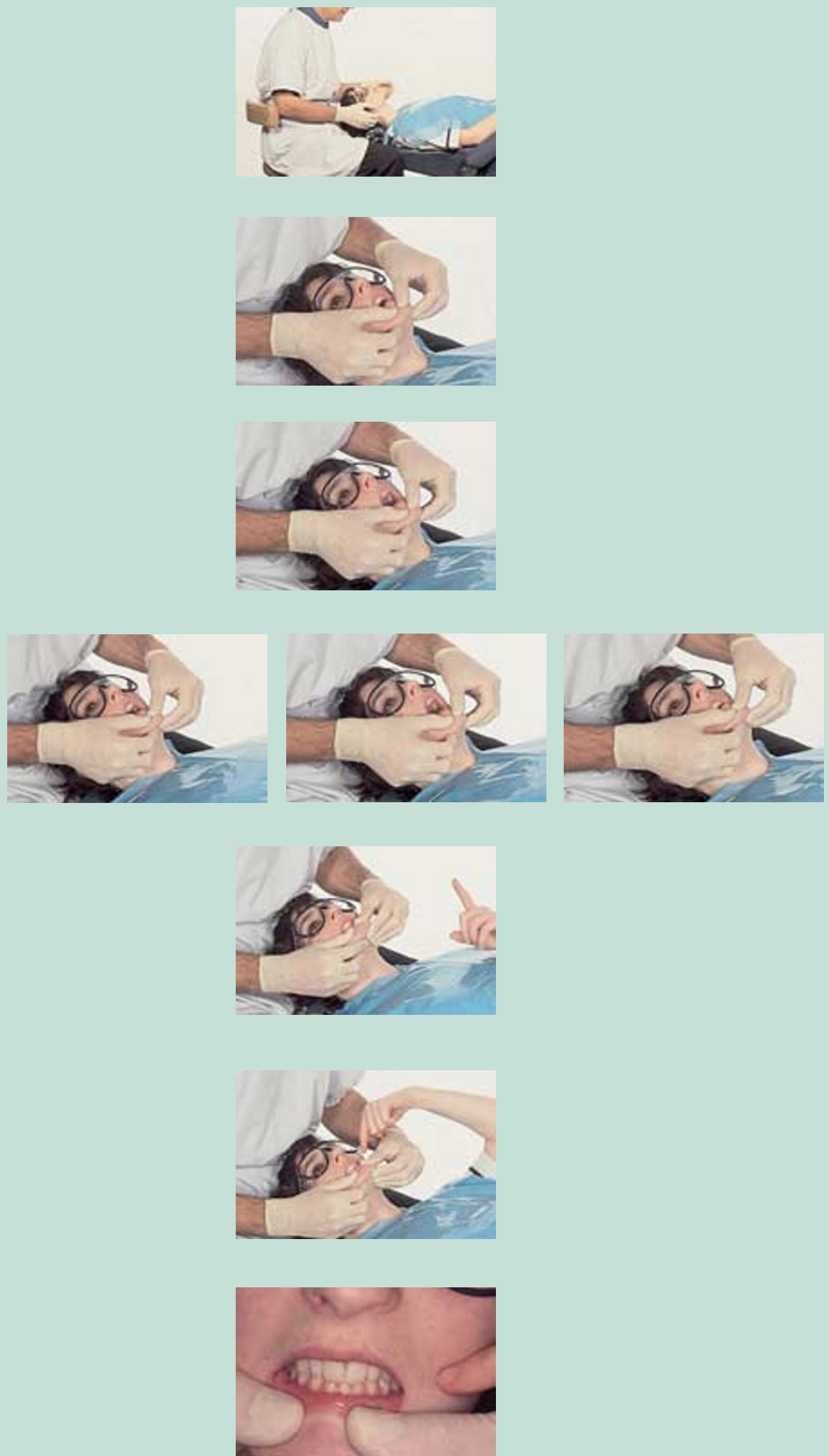

- With chairside assistance use articulating paper or foil to mark premature contact

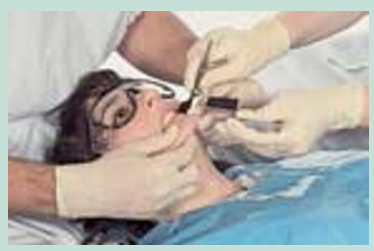


the case this ideal situation does not occur then the Centric Occlusion will not occur in Centric Relation. It will then be possible discover where the premature contact in Centric Relation is (Fig. 6). The positional difference between Centric Relation and Centric Occlusion can further be examined by noting the direction of slide of the mandible when the patient is asked to clench his or her teeth together whilst resting on the Centric Relation premature contact.

If Centric Relation (CR) and Centric Occlusion(CO) do not coincide, in many ways it would make more sense to describe the relationship between the jaws when the teeth are in Centric Occlusion, and not the other way round; but that is impossible to do because there are no landmarks on the jaws that can be examined whilst the patient is holding his or her teeth in Centric Occlusion. So the question has to be: 'Does CO occur in CR?'.

\section{Question 2. Does the patient have Freedom in Centric Occlusion? (Fig. 4)}

This investigation will answer the question: 'Is the patient's Centric Occlusion locked in?' This means when the patient is biting together normally, do his or her incisor teeth prevent those teeth moving slightly forward, or are they prevented from doing so (locked), by the fact that the lower incisor teeth heavily contact the palatal surfaces of the upper incisor teeth?

It can be examined by in one of three ways:

1. Marking the occlusal contacts and seeing if the anterior contacts are heavier than the posterior ones

2. Asking the patient to close together slowly and reporting which teeth hit first

3. Feeling for tremors on the upper incisor teeth with our finger nail whilst the patient repeatedly taps up into Centric Occlusion.

This was easier in the days before routine use of gloves.

\section{Question 3. Where is the patient's Anterior Guidance? (Fig. 5)}

It has already been discussed that the term 'anterior guidance' should not be taken to mean the guidance that is on the front teeth: it is the mandible that is being guided, by the temporomandibular joints (posterior guidance) and by the teeth (anterior guidance). Therefore, which ever teeth touch during excursive movements of the mandible provide the anterior guidance or the dynamic occlusion.

However, the benchmarch against which the patient's occlusion is measured is ideal occlusion (Fig. 7), and in an occlusion which is ideal for the rest of the articulatory system the anterior guidance is on the front teeth. When the anterior guidance is on the back teeth the terminology used is posterior interference. This may be either on the working or non-working side. If interferences are present then they may extend beyond the crossover position. If there are no posterior interferences, then the anterior guidance will be on the front teeth, and this is described as being either 'canine guidance' (where the contact between the upper and lower teeth during an excursive movement of the mandible is against the upper canine and then eventually on the upper central incisors); or it is described as a 'group function' where the anterior guidance is on several teeth. In a group function these contacting teeth are usually the canines and first and second premolars; the more anterior teeth of the group should provide the earlier and harder contacts, otherwise the contact could be considered as a working side interference.

\section{Recording of the occlusion}

It is essential to have a good record of the patient's occlusion if any treatment is to be provided that may have the potential for changing the occlusion. Even if no treatment is envisaged a complete examination of the patient should include the articulatory system of which the occlusion is one of key elements. Just as there are protocols of recording baseline measurements in periodontal and dental disease, there is a need to establish easy and universally reproducible ways of recording the patient's occlusion.

In addition, records are essential for medicolegal reasons.

These records can be either two or three dimensional.

\section{Two dimensional records of the patient's occlusion}

In the main, these rely on marking the static and dynamic occlusal contacts between the teeth and then describing those marks in writing, by diagram or by photograph. In addition to this use of articulating paper or foil to mark the contacts, floss or shimstock can be used to determine whether there is a contact ('pull through' test). The T-scan uses a computer program to analyse the relative hardness of the contacts between the teeth.

With the exception of the T-scan all these two dimensional techniques have the advantage that they are means of determining the occlusal contacts between the actual teeth. They have the disadvantage that is inherent in a two dimensional record of a three dimensional entity.

\section{Three dimensional record of the patient's occlusion}

This, of course, means study models. They have the advantage of being a permanent copy of the patient's occlusion, but the production 
- Finding whether there are Non working side interferences, by trying 'a pull out'

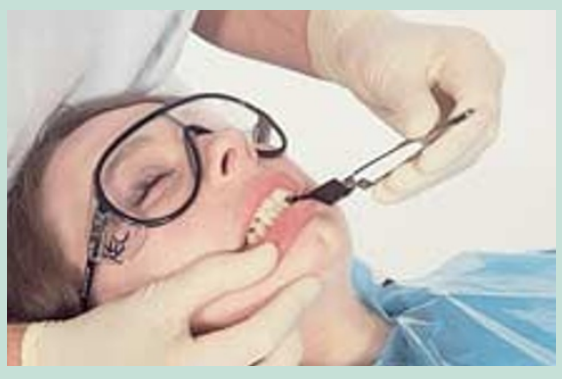

- During this right lateral excursion, there is a NWS interference between UL6 (26) and LL7 (37)

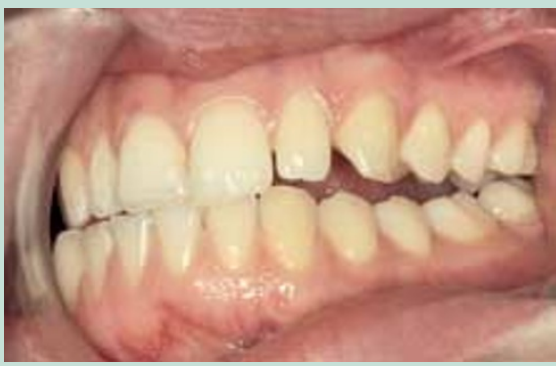

- Examining for the existence of working side interferences by marking the contact between the teeth during a slide to the working side (as illustrated)

- Next mark the centric occlusion stops in a different colour (not illustrated)

- Example of canine guidance

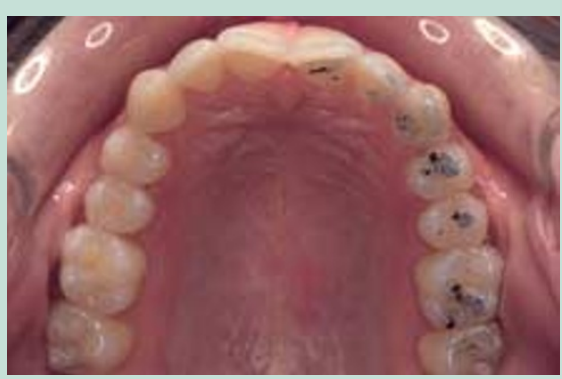

- Example of a group function
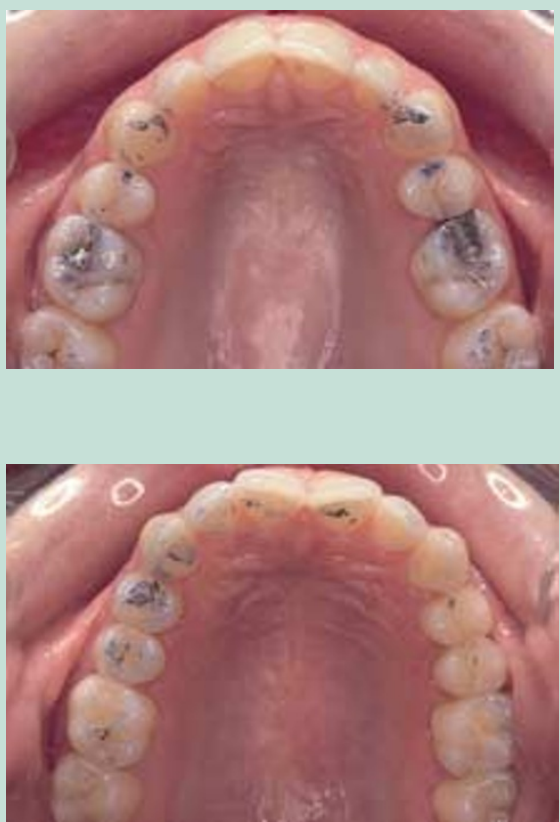
of this copy immediately introduces a host of potential errors. The impressions have to be accurate, the models from those impressions must not only be perfectly cast but also accurately related to each other in a static and dynamic occlusion. This depends not only upon face bows and articulators, but also on the correct use of a range of impression, registration and casting materials.

\section{Discussion}

Study models must be accurate otherwise they might as well not exist; so they need to be verified against either the patient at a check visit or against a second record which could be a simple two dimensional record of the patient's occlusion.

It is therefore the authors' contention that there is a need for a simple reliable two dimensional record of the patient's occlusion and that such a record would be very useful. Consequently these techniques will be illustrated in next section. The advantage of a two dimensional record of the occlusion is that it is not dependent upon a bite registration material, which may introduce error in the model mounting. ${ }^{2}$

\section{Technique}

Articulating paper or foil

If the intention is to mark the occlusal surface of a tooth in order to indicate the position of a contact as opposed to simply discovering whether there is such a contact (shimstock or floss), then a medium to retain some ink needs to be used. It may be double or single sided, but it must be thin and dry (Fig. 9).

At least two colours will be needed. Firstly so that the occlusal contacts in the static and dynamic occlusions can be differentiated; and secondly, so that in operative dentistry the occlusion pre- and post-treatment can be compared.

\section{Articulators}

Articulators are not essential. Their use is not a guarantee of success or of an easy life. Articulators are a very useful tool if the dentist wishes to go in the direction of reproducing the way in which the patient's jaws move one against another. This is valuable information as it assists in the accurate recording of the dynamic occlusion. The nearer a particular articulator can reproduce the patient's movements, the closer it will be possible to construct occlusal schemes that predictably conform to the dentist's objectives, whatever they may be.

In deciding which articulator to use, it is important to ask the question, 'What movements of the mandible do I wish to reproduce, for this patient at this time?'

The key point about this approach is that it

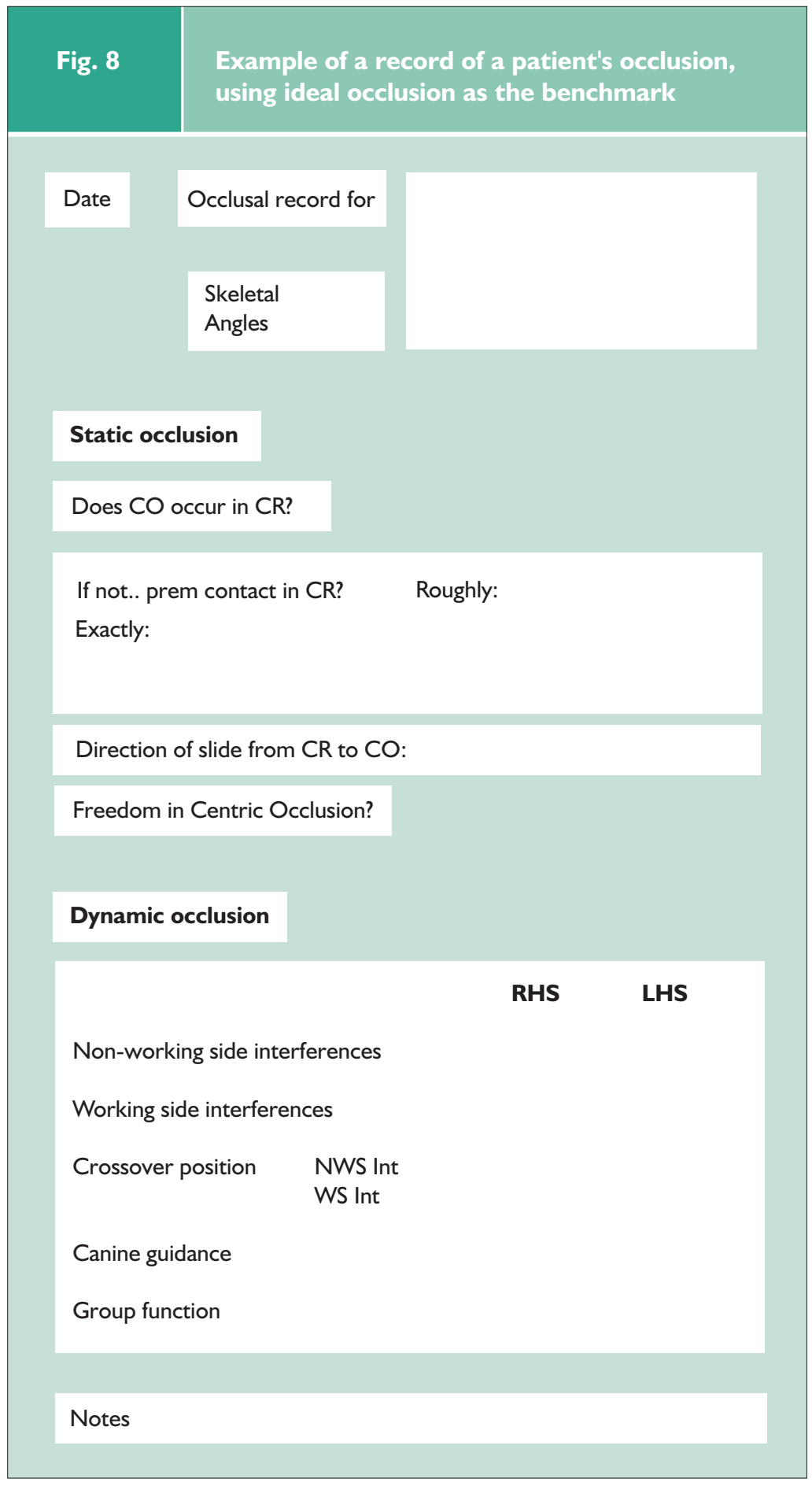

Fig. 9 Paper tissue (for drying occlusal surfaces of
teeth) and two colours of thin $(40 \mu)$
articulating paper (for making occlusal
contacts) held by Miller forceps

Because the paper is thin it is much easier to use it if it is supported by a rigid holder

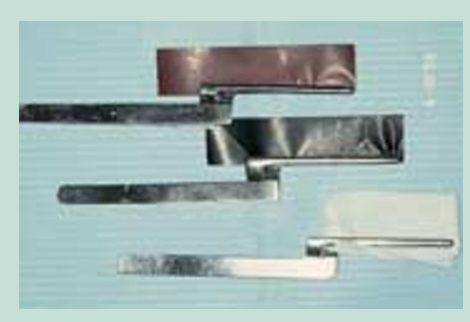


1 Juniper R P. Temporomandibular joint dysfunction; a theory based upon electromyographic studies of the lateral pterygoid muscle. Br J Oral Maxillofac Surg 1984; 22: 1-8.

2 Walls A W G, Wassell R W, Steele J G. A comparison of two methods for locating the intercuspal position (ICP) whilst mounting casts on an articulator. J Oral Rehab 1991; 18 : 43-48

3 Cabot L B. Using articulators to enhance clinical practice. Br Dent $J$ 1998; 184: 272-276.
In the next two sections it will be seen how the examination of the patient's pre-treatment occlusion will be used as the first step in developing good occlusal practice in both simple and advanced restorative dentistry. determines the level of predictability at which the dentist and technician wish to operate. If, as often is the case a dentist and technician are not going to operate with very sophisticated articulators, it does not mean that success is denied to them. It simply means that when checking the occlusion of the restoration it is more likely that adjustment will be necessary. Similarly, if a dentist and technician are operating with sophisticated articulation, it does not remove from the dentist the responsibility of checking the occlusion of the restoration at the fit appointment. A comprehensive overview of articulators can be found elsewhere. ${ }^{3}$

\section{Facebows}

A facebow is a device which enables the maxillary arch to be spatially related to various anatomical landmarks on the patient's face which assists in mounting the maxillary cast within the articulator. The most important consequence of this is that the maxillary cast (in the articulator it is the maxillary member which moves) will bear a similar relationship to the hinges of the articulator as the patient's TMJs does to his or her maxilla, and so to the mandible when a bite registration is used. This matters if the articulator is going to be used for anything other that the static occlusion in centric occlusion.

\section{Guidelines for good occlusal practice}

I. The examination of the patient involves the teeth, periodontal tissues and the articulatory system.

2. There is no such thing as an intrinsically bad occlusal contact, only an intolerable number of times to parafunction on it.

3. The patient's occlusion should be recorded, before any treatment is started.

4. Compare the patient's occlusion against the benchmark of ideal occlusion.

5. A simple, two dimensional means of recording the patient's occlusion before, during and after treatment is an aid to good occlusal practice. 